\title{
Transcriptome Profiling of Oocytes at the Germinal Vesicle Stage from Women from Mongolia with Polycystic Ovary Syndrome
}

\section{Chen Du \\ Xiujuan Chen}

Reproductive Medicine Center, Affiliated Hospital, Inner Mongolia Medical University, Hohhot, Inner Mongolia, 010050, People's Republic of China
Correspondence: Xiujuan Chen Reproductive Medicine Center, Affiliated Hospital, Inner Mongolia Medical

University, I North Pass Street, Hohhot, Inner Mongolia, 01 0050, People's Republic of China

Tel + 86-47I-345I65I

Email chenxh546@163.com
Background: Polycystic ovary syndrome (PCOS) is one of the most common endocrine disorders. Evidence indicates that genetic and environmental factors contribute to the pathogenesis of PCOS. The molecular basis of PCOS is not well understood.

Methods: Whole-genome RNA sequencing was performed on single oocyte at the germinal vesicle (GV) stage from females with normal ovulation and females with PCOS. All subjects were women from Mongolia undergoing intracytoplasmic sperm injection in vitro fertilization (ICSI-IVF) who met the Rotterdam criteria for PCOS. Women with normal ovulation who were undergoing ICSI-IVF owing to male factor infertility were recruited as control subjects.

Results: A total of 1313 differentially expressed genes were found by bio-informatics software in the GV oocytes of PCOS patients and compared with the control group. There were 367 upregulated and 946 downregulated genes (fold change $>2$, false discovery rate $<$ 0.01). When compared with the healthy controls, it was shown that the DEGs like VEGF, IGF, FADS1 et al were investigated as potential causes of PCOS oocytes. The DEGs were related to kinase activity, cell proliferation, gene regulation, and the signaling pathways of phosphatidylinositol 3-kinase, Hippo, and ECM-receptor pathway in patients with PCOS. In addition, the interconnected gene co-expression network was constructed by gene bionetwork analysis, indicating that ITGB5, ITGB3, and CAV2 were the core genes in regulating the module expression of DEGs in PCOS.

Conclusion: RNA sequencing analysis demonstrated DEGs were linked to inflammation, cardiovascular disease, and lipid metabolism in the GV oocytes of women with PCOS. We hypothesize that ITGB5, ITGB3, and CAV2 may be involved in metabolic disorders associated with the different phenotypes of PCOS.

Keywords: polycystic ovarian syndrome, PCOS, RNA-seq, oocyte, transcriptional regulation

\section{Introduction}

Polycystic ovary syndrome (PCOS) is a multifactorial disease associated with endocrine and metabolic disturbances in women of reproductive age and affects both fertility and pregnancy. ${ }^{1,2}$ Defects in oocyte and embryo development are associated with PCOS. ${ }^{3,4}$ Alteration in oocyte competence is considered to be a potential causative factor of subfertility in women with PCOS. ${ }^{5}$ Genetics studies on PCOS have demonstrated that molecular abnormalities, including single-nucleotide polymorphism, global DNA methylation, and dysregulation of microRNA, contribute to the pathogenesis of PCOS. ${ }^{6-9}$ 
PCOS is a risk factor for infertility, and in vitro fertilization (IVF) is recommended as a last resort. It is reported that a high proportion of women with PCOS fail to conceive and may require hospitalization for infertility. ${ }^{10}$ Compared with women without PCOS, the success rate of IVF in women with PCOS has increased by $8-10$ times. After controlling ovarian stimulation treatment, one of the obstacles for patients with PCOS who receive IVF is the insufficient number of mature oocytes, regardless of ovarian response. ${ }^{11-13}$ The clinical pregnancy and live birth rates of females with PCOS who receive IVF are comparable with those of healthy women, but researchers should continue to consider them to be at a high risk of adverse pregnancy-related outcomes. ${ }^{14,15}$ A meta-analysis of the data shows that birth and implantation rates are higher in patients with PCOS than in controls using in vitro maturation. ${ }^{16}$ However, others have been concerned that ARTs may decrease the quality of oocytes and delay the maturation process of oocytes in patients with PCOS. ${ }^{17,18}$ To determine the differentially express genes (DEGs) and potential core regulators of the pathogenesis of PCOS, RNA sequencing (RNA-seq) was performed on oocyte at the germinal vesicle (GV) stage from women with PCOS and women with normal ovulation. Further analysis by gene co-expression network was performed to detect the core genes in regulating the module expression of DEGs in PCOS. This is the first study to identify the specific expression pattern of the GV oocytes of patients with PCOS when compared with the healthy population from Mongolia. The goal of this research is to uncover the foundation of the pathogenesis of PCOS to provide new strategies for diagnosis and targeted therapy at the transcriptional level.

\section{Materials and Methods}

\section{Patient Samples}

We recruited 3 Mongolian women with PCOS and 3 Mongolian women without PCOS, all of whom were undergoing gonadotropin therapy at Affiliated Hospital of Inner Mongolia Medical University, China. Participants (PCOS group; $n=3$ ) were diagnosed with PCOS based on the European society of Human Reproduction and Embryology and the American Society of Reproductive Medicine Consensus Workshop Group. ${ }^{19}$ Patients with Cushing syndrome, late-onset congenital adrenal hyperplasia, thyroid dysfunction, hyperprolactinemia, or androgen-secreting tumors were excluded. Other exclusion criteria included diabetes, hypertension, chronic renal disease, smoking, and the use of alcohol or medications. The inclusion criteria for women with normal ovulation (control group; $\mathrm{n}=3$ ) were a normal menstrual cycle of 21-35d, normal ovulation confirmed by the basal body temperature test, normal ovarian form confirmed by ultrasound, normal androgen levels $(<0.6$ $\mu \mathrm{g} / \mathrm{L})$, a basic level of follicle-stimulating hormone ( $<10 \mathrm{IU} /$ L), and a body mass index of $18.0-25.0 \mathrm{~kg} / \mathrm{cm}^{2}$. Stimulation protocol for all the patients was according to the standard long protocol. There was no significant age difference between the two groups. In addition, all women with normal ovulation underwent intracytoplasmic sperm injection (ICSI) owing to male factor infertility. The study protocol was approved by the Research Ethics committee of Affiliated Hospital of Inner Mongolia Medical University and conducted in accordance with approved institutional guidelines. This study was conducted in accordance with the declaration of Helsinki. All participants gave written informed consent.

\section{Controlled Ovarian Stimulation Protocol}

Gonadotropin releasing hormone agonist (GnRH agonist) 0.1 $\mathrm{mg} / \mathrm{d}$ was used in luteal phase of the last menstrual cycle for pituitary down regulation. After down regulation, the ovulation was induced by recombinant follicle stimulating hormone (rFSH, gonafin, Merck, Serono, Switzerland). The starting dose was 150-225 U. Human menopausal gonadotropin (HMG) was added according to follicular development. When one follicle had a diameter of $18 \mathrm{~mm}$ or two follicles had a diameter of $16 \mathrm{~mm}$, and human chorionic gonadotropin (Lizhu medicine 10,000 IU) was injected. Oocyte retrieval was performed transvaginally $34-36 \mathrm{~h}$ after ovulation was triggered. Cumulus-oocyte complexes were collected in MOPS medium, and the cumulus was mechanically stripped from the oocyte after a brief exposure to hyaluronidase. Oocyte maturation was evaluated by morphological observation. After oocyte maturation, in each group, the mature oocytes (MII phase) were fertilized by ICSI, and the discarded immature GV oocytes were used in this project.

\section{Library Construction and Data Mapping}

The complementary DNA libraries for single-end sequencing were prepared according to the manufacturer's instructions using the Ion Total RNA-seq Kit v2. Before read mapping, clean reads were obtained from the raw reads by removing the adaptor sequences, the reads with $>5 \%$ ambiguous bases (noted as $\mathrm{N}$ ), and low-quality reads. The clean reads were then aligned to the crab-eating macaque genome using the MapSplice program. 


\section{Differentially Expressed Gene Discovery}

We applied the DEseq algorithm to filter the DEGs. Significance and false discovery rate (FDR) were analyzed with the following criteria: $\mid \log 2$ fold change $\mid \geq 2$ (FDR $<$ 0.01 was considered statistically significant). A heat map was drawn based on the $\mathrm{P}$ value, using the differential gene analysis.

\section{Gene Ontology Annotation and Pathway Analysis}

Functional annotations and enrichment tests of genes were carried out using the Database for Annotation, Visualization, and Integrated Discovery (DAVID, http:// david.abcc.ncifcrf.gov) ${ }^{20}$ The significantly enriched Kyoto Encyclopedia of Genes and Genomes (KEGG) pathways in each of the four different gene sets were separately identified against the annotated goat genome based on biological processes, molecular function, and cellular components $(\mathrm{P}<0.05$ was considered to be statistically significant). We then used the KEGG database to build the network of genes according to the relationship among the genes, proteins, and compounds in the database.

\section{Statistical Analysis}

The RNA-seq results for the selected genes, confirmed by real-time quantitative polymerase chain reaction (RT-qPCR), were analyzed using the three tailed $t$-test and the GraphPad Prism 5.0 software (GraphPad, San Diego, CA, USA, www.graphpad.com). The housekeeping gene $\beta$-actin was used as the internal reference. Differences between groups were considered significant if $\mathrm{P}<0.05$.

\section{Result}

RNA-Seq Quality Control and Alignment We performed RNA-seq using single GV oocyte samples from the PCOS and control groups. A total of 510 million high-quality sequences reads were obtained after filtering the raw data. As shown in Table 1, the rate of filtering reads is more than $85 \%$ of the transcripts mapped to the UCSC human genome (http://genome.ucsc.edu). The gene expression level was calculated using the reads per kilobase transcriptome per million mapped reads method.

\section{Discovery of DEGs}

DEGs were determined using DESeq algorithms. Comparisons were made between the GV stage oocytes of the PCOS and the control group. When compared with the control group, a total of 1313 DEGs were found in the GV stage oocytes of the PCOS group. DEGs were visualized by hierarchical clustering and scatterplot (Figure 1). In the PCOS group, 367 genes were upregulated and 946 were downregulated. Vascular endothelial grown factor (VEGF), insulin-like growth factor (IGF), FADS1 which are participants in angiogenesis and lipid metabolism, were significantly increased in oocytes of the PCOS group, while interleukin 6 (IL-6), CXCL1, ADAMTS1, BMP15 and GDF9 which are involved in immune system processes and oocyte development were downregulated. Consistent with the results of other research, we found that the expression of heat shock protein 27 (Hsp27) was markedly decreased in the oocytes of the PCOS group. Our results showed that the expression patterns of the nine key genes analyzed by RT-qPCR agreed with the deep sequencing data, indicating that our deep sequencing data were reliable (Figure 2).

Table I Mapping Statistics of GV Oocytes

\begin{tabular}{|c|c|c|c|c|c|}
\hline Sample & Total Reads Before & Total Reads After & Reads Filter & Reads Mapped & GC\% \\
\hline Controll & $|4,2| 4,544$ & $13,976,138$ & 0.966412 & $85.5 \%$ & 51 \\
\hline Control2 & $12,353,592$ & $11,893,025$ & 0.962717 & $86.3 \%$ & 51 \\
\hline Control3 & $|4,234,52|$ & $13,729,835$ & 0.996834 & $87.1 \%$ & 51 \\
\hline PCOSI & $|I, 84|, 100$ & $11,396,209$ & 0.962428 & $87.2 \%$ & 52 \\
\hline PCOS2 & $|4,52|, 612$ & $13,737,1 \mid 10$ & 0.962437 & $85.8 \%$ & 51 \\
\hline PCOS3 & $15,879,346$ & $14,594,724$ & $0.964 \mid 32$ & $86.2 \%$ & 52 \\
\hline
\end{tabular}

Notes: After obtaining the original data of RNA-seq, the low-quality splices $(\mathrm{Q} \leq 10$ base number and accounting for more than half of the total number of reads) and the reads with more than $3 \%$ of " $\mathrm{N}$ " bases were filtered out, the total number of sequencing reads was mapped into the reference genome by sequence mapping. 


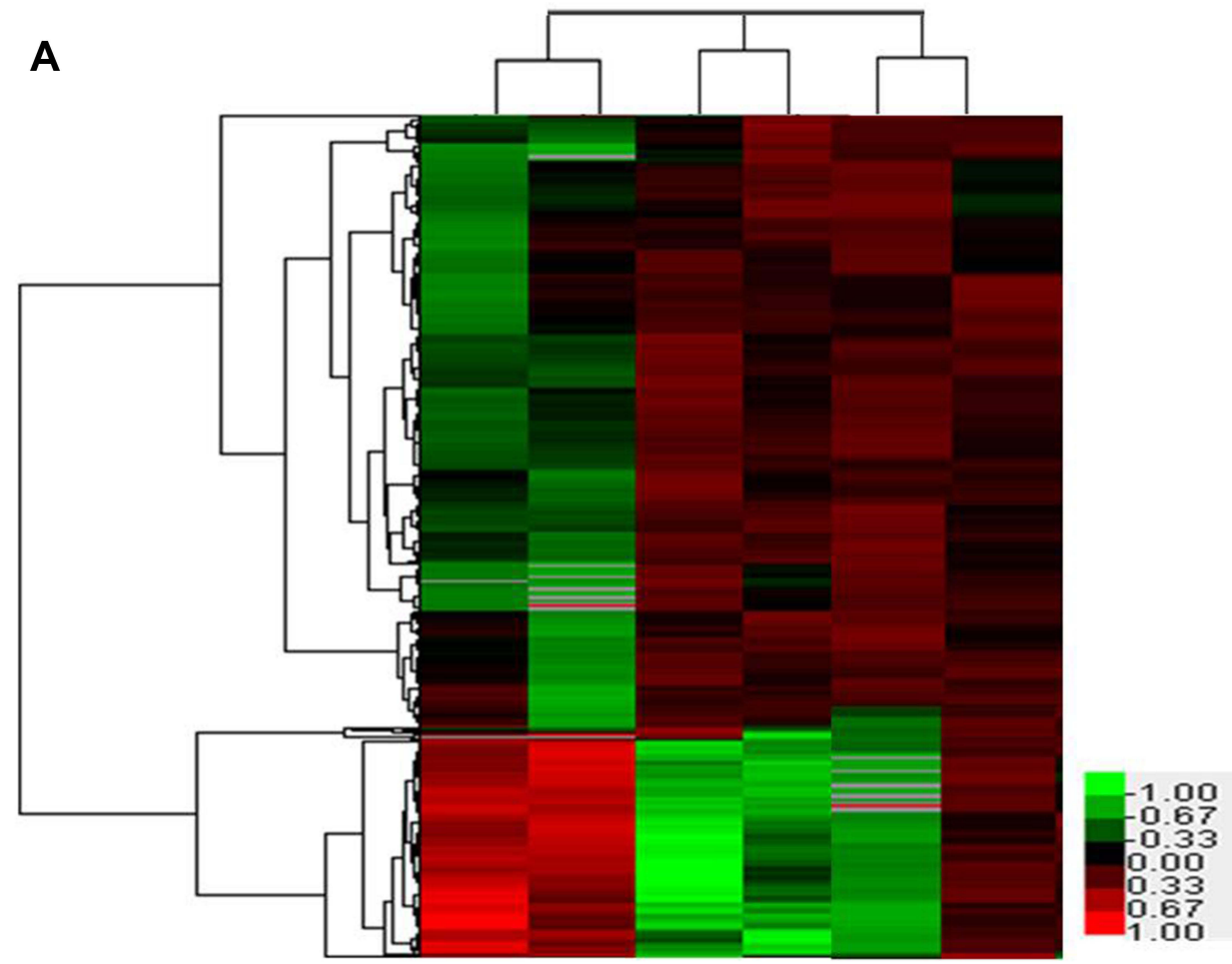

B

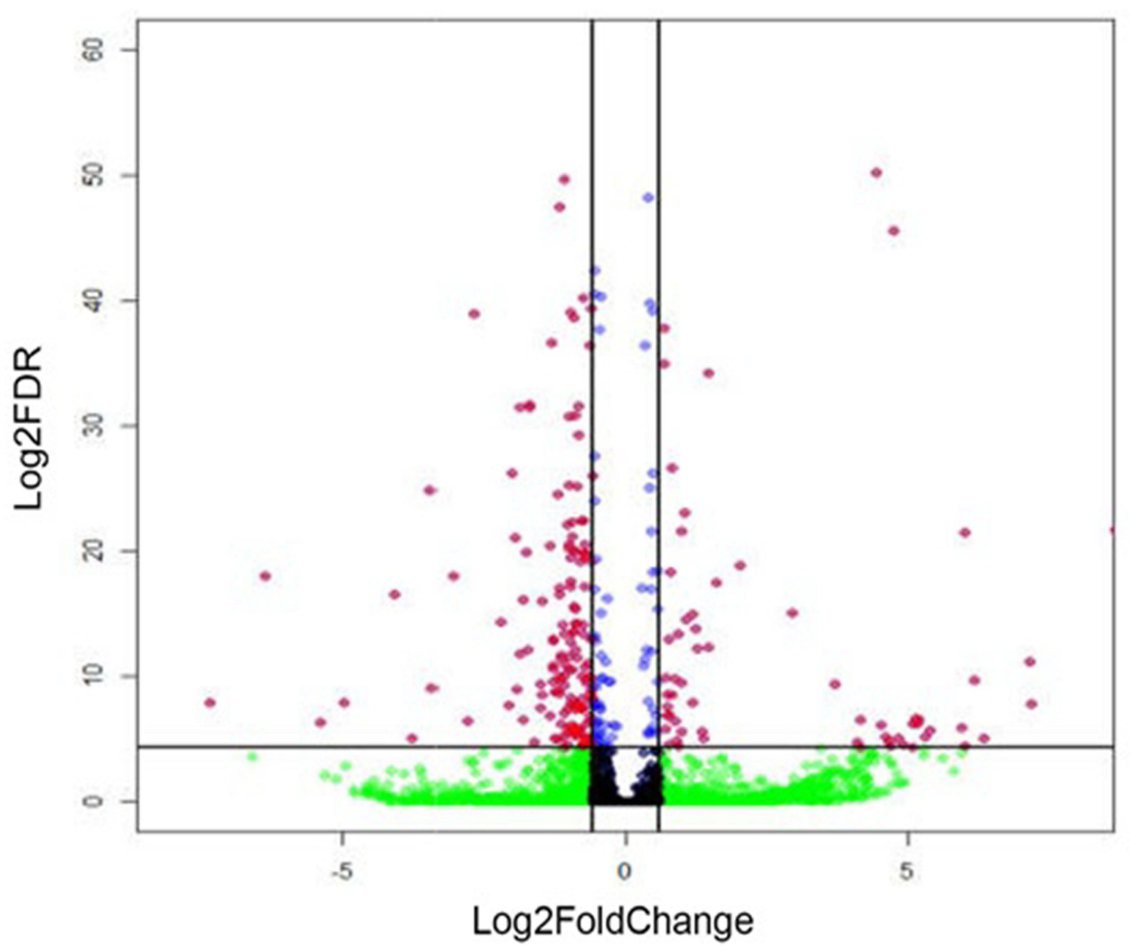

Figure I (A) Heatmap of the I3I3 genes that were differentially expressed between PCOS and control oocyte, (B) Scatterplot (MA plot) of the baseMean of normalized counts versus log2-fold change for all genes generated by DESeq. 


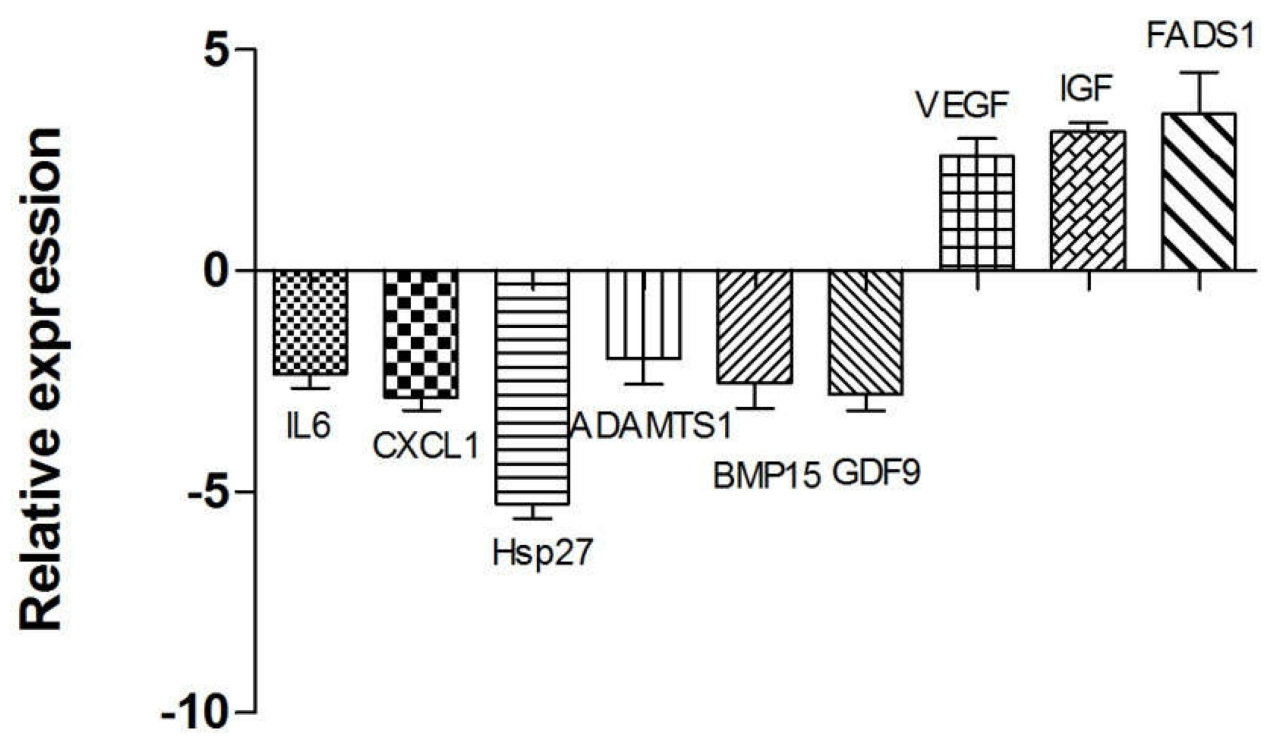

Figure 2 Validation of the expression levels of genes in RNA sequencing by real-time quantitative polymerase chain reaction (RT-qPCR). RT-qPCR gene expression values were determined from the mean of the ratio of $2-\Delta C t$ for the target genes, and the data are expressed as mean \pm standard error of mean (SEM) ( $n=3$ in each group). The $Y$ axis was the difference multiple of PCOS group compared with the control group. Significantly different results for RT-qPCR were determined by one-way ANOVA with Tukey's post-hoc test.

\section{Gene Ontology Cluster Enrichment and Pathway Analysis}

To further investigate the biological processes, three ontologies were selected for annotation. We detected 428 gene ontology (GO) terms, including $288 \mathrm{GO}$ terms in the biological process category, $69 \mathrm{GO}$ terms in the molecular function category, and $71 \mathrm{GO}$ terms in cellular component category. In the molecular function category, the most significantly enriched GO terms were protein binding, kinase activity, and transferase activity (Figure 3). The DEGs were significantly enriched in the cellular components of the extracellular matrix and the proteinaceous extracellular matrix. In the case of biological process, 15 GO terms were significantly enriched and were related to various process, such as cell proliferation and inflammatory response. When comparing the PCOS group with the control group, as shown in Figure 4, 25 significantly enriched pathways ( $\mathrm{P}<0.01)$ were assigned to the DEGs, according to KEGG. Of note, the genes HIF-1, PI3K-Akt, ECM receptor, MAPK, and the Hippo signaling pathway were significantly enriched in the DEGs, possibly contributing to the maturation of oocytes in patients with PCOS.
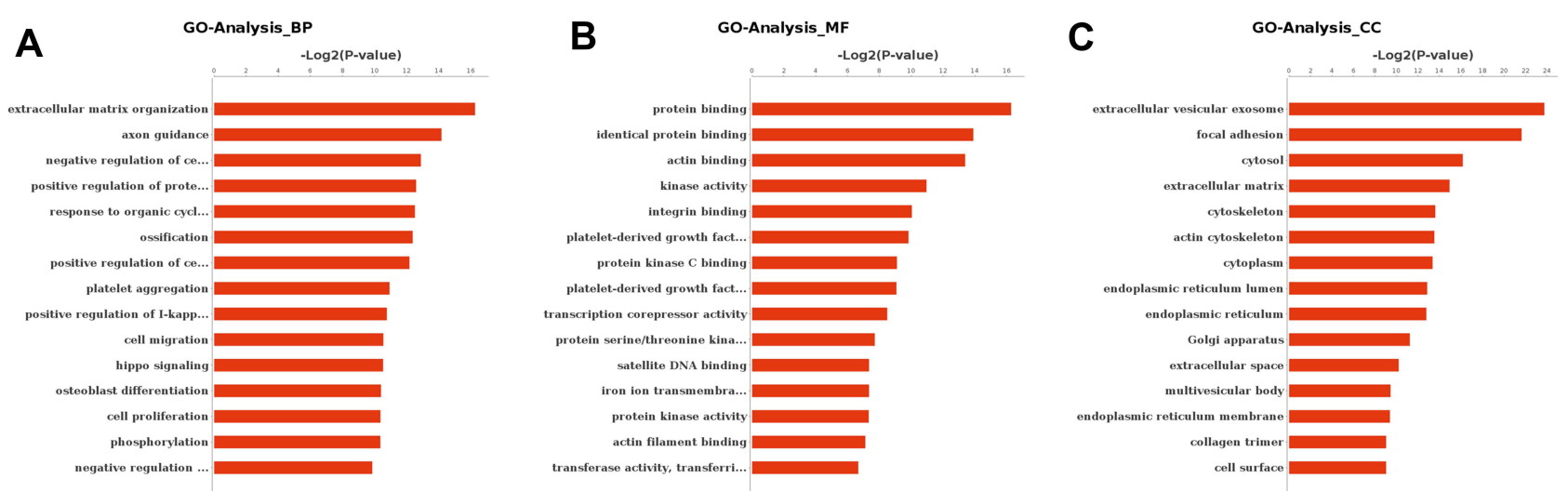

Figure 3 Gene ontology of the differentially expressed genes of oocytes in the germinal vesicle stage. The dataset analyzed was the genes that were differentially regulated (two-fold with FDR P < 0.05) between the PCOS and the control group. In $(\mathbf{A})$ the bar chart represents the proportion of genes that map to a gene ontology term associated with a biological process. In (B) the bar chart represents the proportion of genes that map to a gene ontology term associated with a cellular component. In (C) the bar chart represents the proportion of genes that map to a gene ontology term associated with a cellular component molecular function. 


\section{Pathway-Analysis}

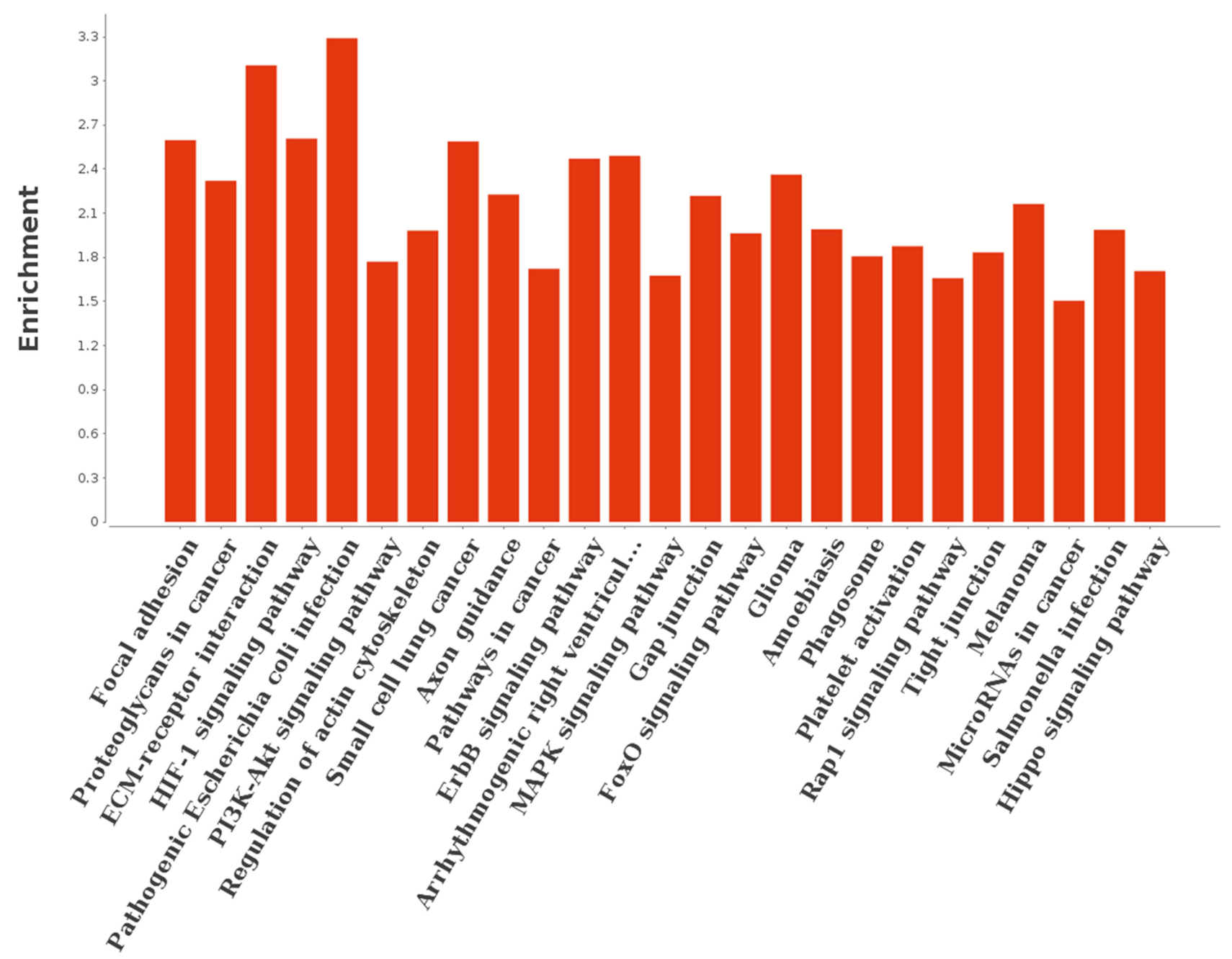

Figure 4 KEGG classification of the differentially expressed genes of oocytes in the germinal vesicle stage.

\section{Differential Gene Network Map}

To determine the potential core regulators in PCOS oocytes, a bionetwork analysis of oocytes in the GV stage was performed. As shown in Figure 5, there were many interactive partners of the ITGB5, ITGB3, and CAV2 genes that played a central role in the co-expression network. Our network for this module identified that ITGB5, ITGB3, and CAV2 genes expressed in oocytes presented the highest degrees of connectivity. These genes are enriched in the PI3K/Akt signaling pathway, which can regulate the meiotic events of oocytes and affect the maturation ability of oocytes, indicating that PI3K/Akt signaling pathway is involved in the regulation Oocyte maturation.

\section{Discussion}

Although much progress has been made in recent years, oocyte development incompetence, implantation failure, and pregnancy loss have remained the main issues facing patients with PCOS undergoing IVF. ${ }^{21}$ Failure to select a dominant follicle and the number of arrested and atretic follicles are significantly increased in polycystic ovaries. ${ }^{22}$ The oocyte is rich in cytoplasm that contains yolk granules to support the cell's growth, maturation, and early development after fertilization. The goal of our study is to better understand the molecular mechanisms underlying altered fertility in patients with PCOS and to provide potential therapeutic targets for treatment.

Previous studies demonstrated that endocrine hormones, such as luteinizing hormone and testosterone, may be involved in abnormal follicular genesis in the ovaries of patients with PCOS. ${ }^{23,24}$ Patients with PCOS undergoing ovarian stimulation for IVF have an increased risk of impaired oocyte developmental competence, 


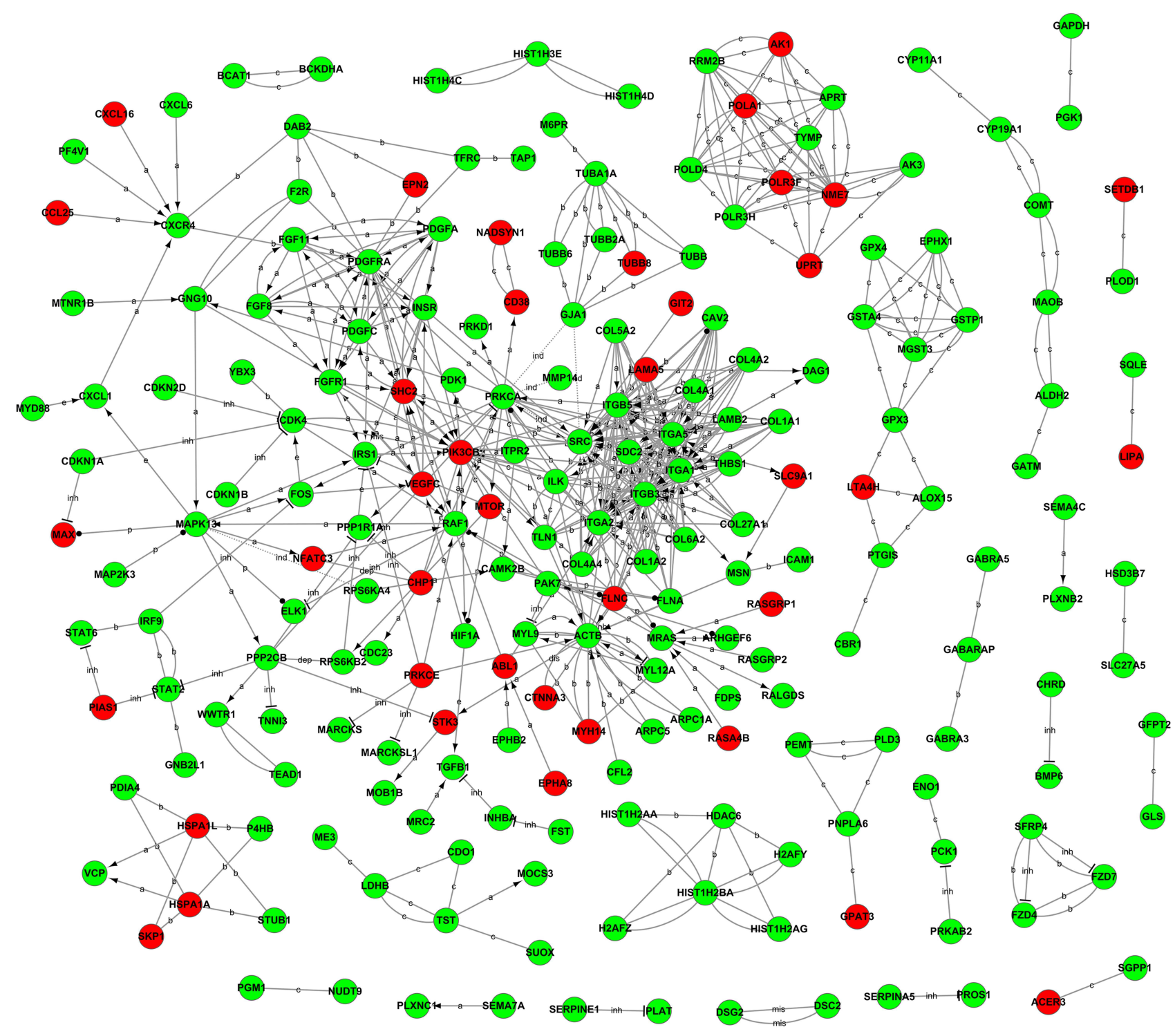

Figure 5 Gene network of known relationships between those differentially expressed genes. The network was generated in the PCOS group using triangle connectivity based on focus genes and built up according to the number of interactions between a single prospective gene and others in the existing network and the number of interactions the prospective gene has with other genes outside this network.

implantation failure, and pregnancy loss. The current study used a system approach to detect all genes that are differentially expressed in response to several different growth factors and hormone treatments known to influence the maturation of oocytes. ${ }^{25}$ RNA-Seq has been widely used to identify DEGs when comparing patients with healthy controls. This research presents, for the first time, a complete dataset comparing oocytes at the GV stage, using RNA-seq, of women with PCOS and those with normal ovulation. Our data indicate that some differentially expressed genes play important roles in mediating meiosis, hormone receptor signaling, intercellular communication, proliferation, follicular formation, DNA repair and oxidative stress. Hsp27 in GV oocytes was four-fold lower the PCOS group than in the control group, which is consistent with results reported by Cai et al. ${ }^{26}$ It is thought that Hsp27 can decrease the maturation rate of oocytes derived from patients with PCOS. Several studies have found that the early stage of apoptosis is closely related to oocyte development. Therefore, the reduced expression of GDF9 and BMP15 in oocytes may have detrimental effects on follicular development in the ovaries of patients with PCOS. These findings indicate that aberrant expression of oocyte secretion factors might be involved in the aberrant follicular development associated with PCOS. $^{27} \mathrm{~A}$ distintegrin and metalloprotease with 
thrombospondin type 1 motifs (ADAMTS-1) is involved in normal folliculogenesis and the ovulatory process, and it plays an important role in female fertility. Several researchers have found that ADAMTS-1 decreases in patients with PCOS and is strongly associated with impaired oocyte quality. ${ }^{28}$ Lipid metabolism genes associated with insulin resistance, such as FADS1, are significantly upregulated. Our study confirmed that genes are differentially expressed in women with PCOS when compared with the control group. Genes associated with immunity are also differentially expressed in PCOS, including IL-6 and CXCL1, which enhance follicular assembly.

The GO project is a collaborative effort to develop and use ontologies to support the biological annotation of genes and their products. ${ }^{29}$ In this work, we performed a GO analysis to describe the properties of DEGs in PCOS oocytes and screened for significant GO terms $(\mathrm{P}<0.05)$. Coordinated regulation of signaling pathways is crucial for normal embryonic development and the establishment of pregnancy. In our study, the DEG genes in oocytes at the GV stage were included in the MAPK signaling pathway, MAPK signaling, TGF- $\beta$ signaling pathway, VEGF signaling pathway, and PI3K-Akt signaling pathway. We observed 30 DEGs (RPS6KA4, STK4, FLNA, MAX, FGF8, RELB, STK3, RAF1, MRAS, ELK1, TGFB1, PDGFRA, RASGRP1, MKNK2, MAP2K3, MAPK8IP1, FLNC, CD14, MAPK13, FGFR1, RASGRP2, FGF11, GADD45B, HSPA1L, CHP1, HSPA1A, FOS, PRKCA, PDGFA, and NFATC3) in the MAPK signaling pathway. The MAPK is responsible for a variety of processes, including the transcriptional activation of cytokines, chemokines, and other inflammatory mediators. ${ }^{30}$ The Hippo pathway also plays an important role in restricting organ size by regulating proliferation, apoptosis, follicle-cell differentiation, and oocyte polarity. ${ }^{31}$ The PI3K-Akt signaling pathway participates in COCs development in women with PCOS because it is related to hyperandrogenism, insulin resistance, inflammation, and oxidative stress. Extracellular matrix (ECM) is a complex mixture of structural and functional macromolecules, which plays an important role in cell and tissue structure, function maintenance, tissue and organ morphogenesis.

In addition, to determine the key genes in the regulation of the maturation of oocytes, we constructed a network with putative regulatory connections to neighbors. Examination of these networks can yield inferences about how genes interact to regulate oocyte development and can identify potentially important control points within the regulatory networks of the oocyte. When the list of genes that were found to be differentially expressed during follicle assembly was analyzed, it was discovered that the genes ITGB5, ITGB3, and CAV2 had the most regulatory connections. Our analysis suggests that these genes are key in the regulation of oocyte development and maturation. The possible role of these genes in the fertility of Mongolian women will need more studies. The limitation of this study is that the sample size is too small. In the future experiments, it is necessary to increase the sample size for further study.

\section{Conclusion}

This study is the first time to use high-throughput RNAseq technology to sequence the transcriptome of single oocyte in GV stage of Mongolian PCOS patients and normal ovulating women, reveal the expression characteristics of immature oocytes, and obtain genes with important functions in the expression process of oocytes in GV stage of PCOS patients. We identified genes associated with cell-cell communication, proliferation, hormone receptor signaling, folliculogenesis, and development in PCOS oocytes. MAPK signaling pathway, PI3K-Akt signaling pathway, ECM-receptor pathway, Hippo signaling pathway may play a vital role in the development of oocytes, because most of the differentially expressed genes we identified were involved in these pathways. ARTs can be an effective treatment for subfertile patients with PCOS.

\section{Acknowledgments}

The members from department of Obstetrics and Gynecology in Inner Mongolia Medical University assisted in the collection and processing of the data.

\section{Funding}

The authors are grateful for support by grants from Inner Mongolia Autonomous Region (IMAR), Natural Science Foundation (2015BS0802; 2019MS08121), Inner Mongolia Education Department Foundation (NJZY19104), and Inner Mongolia Medical University Affiliated Hospital Doctor Launches Foundation Program (NYFYBS201602).

\section{Disclosure}

The authors report no conflicts of interest in this work. 


\section{References}

1. Ntumy M, Maya E, Lizneva D, et al. The pressing need for standardization.in epidemiologic studies of PCOS across the globe. Gynecol Endocrinol. 2019;35:1-3. doi:10.1080/09513590.2018.1488958

2. Olaniyi KS, Oniyide AA, Adeyanju OA, et al. Low dose spironolactone-mediated androgen-adiponectin modulation alleviates endocrine-metabolic disturbances in letrozole-induced PCOS. Toxicol Appl Pharmacol. 2020;411:115381. doi:10.1016/j.taap.2020.115381

3. Deepika K, Sindhuma D, Kiran B, et al. Empty follicle syndrome following GnRHa trigger in PCOS patients undergoing IVF cycles. $J$ Reprod Fertil. 2018;19(1):16-25.

4. Widecka J, Ozegowska K, Banaszewska B, et al. Is copeptin a new potential biomarker of insulin resistance in polycystic ovary syndrome? Ginekol Pol. 2019;90(3):115-121. doi:10.5603/GP.2019.0021

5. Palomba S, Daolio J, Sala GB. Oocyte competence in women with polycystic ovary syndrome. Trends Endocrinol Metab. 2017;28 (3):186-198. doi:10.1016/j.tem.2016.11.008

6. Krentowska A, Ebkowska A, Jacewicz-Wicka M, et al. Metabolic syndrome and the risk of cardiovascular complications in young patients with different phenotypes of polycystic ovary syndrome. Endocrine. 2021;5:1-11.

7. Wang F, Niu WB, Kong HJ, et al. The role of AMH and its receptor SNP in the pathogenesis of PCOS. Mol Cell Endocrinol. 2017;43 (9):363-368. doi:10.1016/j.mce.2016.09.023

8. Murri M, Insenser M, Fernández-Durán E, et al. Non-targeted profiling of circulating microRNAs in women with polycystic ovary syndrome (PCOS): effects of obesity and sex hormones. Metab Clin Exp. 2018;86:49-60.

9. Vázquez-Martínez ER, Gómez-Viais YI, García-Gómez E, et al. DNA methylation in the pathogenesis of polycystic ovary syndrome. Reproduction. 2019;158(1):27-40. doi:10.1530/REP-18-0449

10. Azziz R. Polycystic ovary syndrome. Obstet Gynecol. 2018;132 (2):321-336. doi:10.1097/AOG.0000000000002698

11. Cordeiro FB, Cataldi TR, de Souza BZ, et al. Hyper response to ovarian stimulation affects the follicular fluid metabolomic profile of women undergoing IVF similarly to polycystic ovary syndrome. Metabolomics. 2018;14(4):1-11. doi:10.1007/s11306-018-1350-z

12. Sánchez F, Lolicato F, Romero $S$, et al. An improved ivm method for cumulus-oocyte complexes from small follicles in polycystic ovary syndrome patients enhances oocyte competence and embryo yield. Hum Reprod. 2017;32(10):2056-2068. doi:10.1093/humrep/ $\operatorname{dex} 262$

13. Yang ZY, Chian RC. Development of in vitro maturation techniques for clinical applications. Fertil Steril. 2017;108(4):577-584. doi:10.1016/j.fertnstert.2017.08.020

14. Teede HJ, Misso ML, Costello MF, et al. Recommendations from the international evidence-based guideline for the assessment and management of polycystic ovary syndrome. Clin Endocrinol. 2018;89 (3):251-268. doi:10.1111/cen.13795

15. Jo J, Lee YJ. Effectiveness of acupuncture in women with polycystic ovarian syndrome undergoing in vitro fertilisation or intracytoplasmic sperm injection: a systematic review and meta-analysis. Acupunct Med. 2017;35(3):162. doi:10.1136/acupmed-2016-011163

16. Abedini M, Ghaedi E, Hadi A, et al. Zinc status and polycystic ovarian syndrome: a systematic review and meta-analysis. $J$ Trace Elem Med Biol. 2019;52:216-221. doi:10.1016/j.jtemb.2019.01.002
17. Sigala J, Sifer C, Dewailly D, et al. Is polycystic ovarian morphology related to a poor oocyte quality after controlled ovarian hyperstimulation for intracytoplasmic sperm injection? Results from a prospective, comparative study. Fertil Steril. 2015;103(1):112-118. doi:10.1016/j.fertnstert.2014.09.040

18. Liu Q, Li Y, Feng Y, et al. Single-cell analysis of differences in transcriptomic profiles of oocytes and cumulus cells at GV, MI, MII stages from PCOS patients. Sci Rep. 2016;6(1):39638. doi:10.1038/ srep39638

19. The Rotterdam ESHRE/ASRM-sponsored PCOS consensus workshop group. Revised 2003 consensus on diagnostic criteria and long-term health risks related to polycystic ovary syndrome (PCOS). Fertil Steril. 2004;81(1):19-25. doi:10.1016/j.fertnstert.2003.10.004

20. Huang DW, Sherman BT, Lempicki RA. Bioinformatics enrichment tools: paths toward the comprehensive functional analysis of large gene lists. Nucleic Acids Res. 2019;37:1-13. doi:10.1093/nar/gkn923

21. Altmäe S, Mendoza-Tesarik R, Mendoza C, et al. Effect of growth hormone on uterine receptivity in women with RIF in an oocyte donation program: a randomized controlled trial. J Endocr Soc. 2018;2(1):96-105. doi:10.1210/js.2017-00359

22. Liu J, Wu DC, Qu LH, et al. The role of mtor in ovarian cancer, polycystic ovary syndrome and ovarian aging. Clin Anat. 2018;31:891-898. doi:10.1002/ca.23211

23. Emekci OO, Ozay AC, Acar B, et al. Role of kisspeptin in polycystic ovary syndrome (PCOS). Gynecol Endocrinol. 2016;32(9):1-5.

24. Gupta M, Yadav R, Mahey R, et al. Correlation of body mass index (BMI), anti-mullerian hormone (AMH), and insulin resistance among different polycystic ovary syndrome (PCOS) phenotypes - a crosssectional study. Gynecol Endocrinol. 2019;35(11):970-973. doi:10.1080/09513590.2019.1613640

25. Nilsson E, Zhang B, Skinner MK. Gene bionetworks that regulate ovarian primordial follicle assembly. BMC Genom. 2013;14(1):496. doi:10.1186/1471-2164-14-496

26. Cai L, Ma X, Liu S, et al. Effects of upregulation of Hsp27 expression on oocyte development and maturation derived from polycystic ovary syndrome. PLoS One. 2013;8(12):e83402. doi:10.1371/journal. pone. 0083402

27. Wei LN, Huang R, Li LL, et al. Reduced and delayed expression of GDF9 and BMP15 in ovarian tissues from women with polycystic ovary syndrome. J Assist Reprod Genet. 2014;31(11):1483-1490. doi:10.1007/s10815-014-0319-8

28. Karakose M, Demircan K, Tutal E, et al. Clinical significance of ADAMTS1, ADAMTS5, ADAMTS9 aggrecanases and IL-17A, IL23, IL-33 cytokines in polycystic ovary syndrome. J Endocrinol Invest. 2016;39(11):1-7. doi:10.1007/s40618-016-0472-2

29. Shen H, Liang Z, Zheng S, et al. Pathway and network-based analysis of genome wide association studies and rt-pcr validation in polycystic ovary syndrome. Int J Mol Med. 2017;40(5):1385-1396. doi:10.3892/ ijmm.2017.3146

30. Koga Y, Tsurumaki H, Aoki-Saito H, et al. Roles of cyclic AMP response element binding activation in the ERK1/2 and p38 MAPK signalling pathway in central nervous system, cardiovascular system, osteoclast differentiation and mucin and cytokine production. Int $J$ Mol Sci. 2019;20(6):1346. doi:10.3390/ijms20061346

31. Negron VM, Hansen PJ. Role of yes-associated protein 1, angiomotin, and mitogen-activated kinase kinase $1 / 2$ in development of the bovine blastocyst. Biol Reprod. 2018;98(2):170-183. doi:10.1093/ biolre/iox172 


\section{Publish your work in this journal}

The International Journal of General Medicine is an international, peer-reviewed open-access journal that focuses on general and internal medicine, pathogenesis, epidemiology, diagnosis, monitoring and treatment protocols. The journal is characterized by the rapid reporting of reviews, original research and clinical studies across all disease areas. The manuscript management system is completely online and includes a very quick and fair peer-review system, which is all easy to use. Visit http://www.dovepress.com/ testimonials.php to read real quotes from published authors. 